\title{
ON THE PACKING CHROMATIC NUMBER OF SEMIREGULAR POLYHEDRA
}

\author{
Marián KLEŠČ, Štefan SCHRÖTTER \\ Department of Mathematics and Theoretical Informatics, Faculty of Electrical Engineering and Informatics, Technical University of \\ Košice, Letná 9, 04200 Košice, Slovak Republic, e-mail: marian.klesc@tuke.sk, stefan.schrotter@tuke.sk
}

\begin{abstract}
Packing colouring of a graph $G$ is a partitioning of the vertex set of $G$ with the property that vertices in i-th class have pairwise distance greater than $i$. The packing chromatic number of $G$ is the smallest integer $k$ such that the vertex set of $G$ can be partitioned as $X_{1}, X_{2}, \ldots, X_{k}$ where $X_{i}$ is an i-packing for each $i$. In the paper, the packing chromatic numbers for all Platonic solids as well as for all prisms are given.
\end{abstract}

Keywords: graph, polyhedron, Platonic solid, packing chromatic number

\section{INTRODUCTION}

The concept of packing colouring comes from the area of frequency planning in wireless networks. This model emphasizes the fact that some frequencies might be used more sparely than the others. In graph terms, we ask for a partitioning of the vertex set of a graph $G$ into disjoint classes. Let $G=(V, E)$ be a graph with vertex set $V(G)$ and edge set $E(G)$ and let $d(u, v)$ denote the distance between vertices $u$ and $v$ in $G$. A packing $k$-colouring of a graph $G$ is a mapping $c: V(G) \rightarrow\{1,2, \ldots, k\}$ such that any two vertices $u$ and $v$ of a colour $i$ satisfies $d(u, v)>i$. Thus, the vertices of $G$ are partitioned into different colour classes $X_{1}, X_{2}, \ldots, X_{k}$, where every $X_{i}$ is an $i$-packing of $G$. The $i$ packing number of $G$, denoted by $\rho_{i}(G)$, is the maximum cardinality of an $i$-packing that occurs in $G$. The smallest integer $k$ for which there exists a packing $k$-colouring of $G$ is called the packing chromatic number of $G$ and it is denoted by $\chi_{p}(G)$. The notion of the packing chromatic number was established by Goddard et al. [3] under the name broadcast chromatic number. The term packing chromatic number was introduced by Brešar et al. [2]. The determination of the packing chromatic number is computationaly difficult. It was shown to be $N P$-complete for general graphs in [3]. Fiala and Golovach [5] showed that the problem remains $N P$-complete even for trees. Recently, some other aspects concerning the packing chromatic number of graphs were studied in [4] and [6].

A polyhedron is a geometric solid in three dimensions with flat faces and straight edges. A polyhedron $P$ is called semiregular if all its faces are regular polygons and there exists a sequence $\sigma=\left(p_{1}, p_{2}, \ldots, p_{q}\right)$ such that every vertex of $P$ is surrounded by a $p_{1}$-gon, a $p_{2}$-gon, $\ldots$, a $p_{q}$-gon, in this order within rotation and reflection. A semiregular polyhedron $P$ is called the $\left(p_{1}, p_{2}, \ldots, p_{q}\right)$-polyhedron if it is determined by the cyclic sequence $\sigma=\left(p_{1}, p_{2}, \ldots, p_{q}\right)$. The set of semiregular polyhedra consists of precisely five Platonic solids, thirteen Archimedean solids, a single $(3,4,4,4)$ - polyhedron discovered by Ashkinuze [1], and two infinite families: the prisms, i.e. $(4,4, n)$-polyhedra for every $n \geq 3, n \neq 4$, and the antiprisms, i.e. $(3,3,3, n)$ polyhedra for every $n \geq 4$. The problems of colouring vertices, edges or faces of semiregular polyhedra were studied from several aspects, see for example [7]. The main purpose of this paper is to determine the packing chromatic number of all Platonic solids and of the infinite family of prisms.

Instead of studying convex polyhedra it is enough to study their graphs, i.e. graphs determined by vertices and edges of polyhedra. This is allowed due to a famous theorem given by Steinitz that states that a graph is the graph of a convex polyhedra if and only if it is planar and 3-connected. The diameter, $\operatorname{diam}(G)$, of a graph $G$ is the maximum distance between any two vertices of $G$. For a vertex $x \in V(G)$, by $N_{i}[x]$ we denote the set $\{u \in V(G) \mid d(u, x) \leq i\}$.

\section{PLATONIC SOLIDS}

The set of Platonic solids consists of five members: (i) the tetrahedron, or equivalently, the (3,3,3)-polyhedron,

(ii) the octahedron, or the $(3,3,3,3)$-polyhedron,

(iii) the cube, i.e. the $(4,4,4)$-polyhedron,

(iv) the icosahedron, i.e. the $(3,3,3,3,3)$-polyhedron,

(v) the dodecahedron, i.e. the $(5,5,5)$-polyhedron.

\subsection{The tetrahedron}

The tetrahedron consists of four vertices and also of four triangular faces. The graph of the tetrahedron is $K_{4}$, the complete graph on four vertices. It immediately implies that the following is true:

Theorem 2.1. Let $T$ be the tetrahedron. Then $\chi_{p}(T)=4$.

\subsection{The octahedron}

The octahedron $O$ consists of six vertices and of eight triangular faces. In the plane, it is represented by the regular graph with every vertex of degree four. As the diameter of the graph $O$ is two, the subset $X_{1}$ of $V(O)$ containing the vertices labelled by the colour 1 can contain at most two elements. Hence, $\rho_{1}(O) \leq 2$. Every other colour can be used only once and therefore, $\chi_{p}(O) \geq 5$. The reader can easy verify that there is a packing 5-colouring of the graph $O$. This immediately implies:

Theorem 2.2. Let $O$ be the octahedron. Then $\chi_{p}(O)=5$. 


\subsection{The cube}

In the plane, the cube $Q$ is represented by the graph shown in Fig. 11 The diameter of the graph $Q$ is three, which implies that $\rho_{2}(Q)=2$ and $\rho_{i}(Q) \leq 1$ for all integer $i \geq 3$. In Fig. 1 one can easily verify that $\rho_{1}(Q)=4$. In the graph $Q$ there are four pairs of vertices in distance three. Every such pair has one vertex labelled by 1 , which implies that for $\left|X_{1}\right|=\rho_{1}(Q)=4$ we have $\left|X_{2}\right|=1<\rho_{2}(Q)$. Similarly, for $\left|X_{2}\right|=\rho_{2}(Q)=2$ we have $\left|X_{1}\right| \leq 3$. Thus, as $\left|X_{1} \cup X_{2}\right|=5$, in a packing $k$-colouring of $Q$, at least three other colours are necessary and $\chi_{p}(Q) \geq 5$. As there is a packing 5-colouring of the graph $Q$, the next result is obvious.

Theorem 2.3. Let $Q$ be the cube. Then $\chi_{p}(Q)=5$.

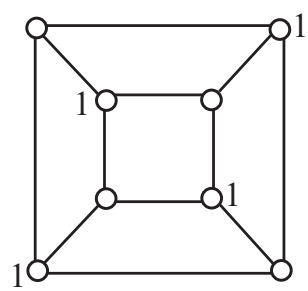

Fig. 1 A drawing of $Q$ with $\rho_{1}(Q)=4$

\subsection{The icosahedron}

The graph $I$ of the icosahedron is drawn in Fig. 2 Its diameter is three, thus $\rho_{2}(I)=2$ and $\rho_{i}(I) \leq 1$ for all integer $i \geq 3$. Due to symmetry, if one vertex of $I$ is labelled by the colour 1 , then the six vertices in distance more than 1 from this vertex form a subgraph of $I$ which is isomorphic to the wheel $W_{5}$. In this case, at most two of these six considered vertices can be labelled by the colour 1 , because $\rho_{1}\left(W_{5}\right)=2$. So, $\rho_{1}(I)=3$. There is a packing $k$-colouring of the graph $I$ with $\left|X_{1}\right|=3$ and $\left|X_{2}\right|=2$, see Fig. 2. As for every of the other seven vertices one new colour is necessary, $\chi_{p}(I)=2+7$.

Theorem 2.4. Let I be the icosahedron. Then $\chi_{p}(I)=9$.

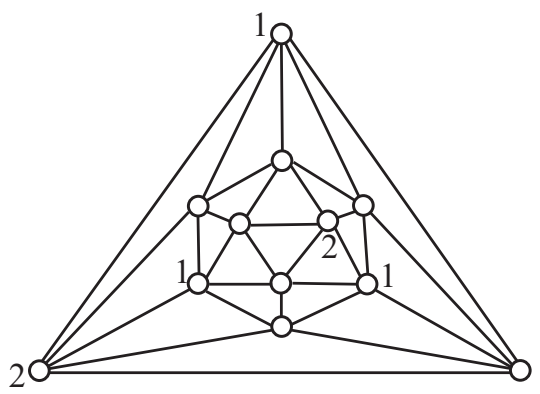

Fig. 2 A graph of the icosahedron

\subsection{The dodecahedron}

Theorem 2.5. For the dodecahedron $D, \chi_{p}(D)=9$.

Proof. In Fig. 3 there is the graph $D$ of the dodecahedron. As $\operatorname{diam}(D)=5, \rho_{i}(D) \leq 1$ for all integer $i \geq 5$. Consider an arbitrary vertex $x$ of the graph $D$. Only one vertex of $D$ is not contained in $N_{4}[x]$. Thus, $\rho_{4}(D)=2$. Similarly, $N_{3}[x]$ contains all vertices of $D$ except of four vertices which induce the subgraph $K_{1,3}$ of $D$. This forces $\rho_{3}(D)=2$. Every face of the dodecahedron is bounded by a cycle of length five and at most one vertex of a cycle of length five can obtain the colour 2. Moreover, every vertex is incident with exactly three faces. Thus, at most $\frac{12}{3}=4$ vertices can obtain the colour 2. It is easy to find an assignment with four colours 2 and therefore, $\rho_{2}(D)=4$. The colour 1 can appear on the boundary of every face only twice. As every vertex is incident with three faces, at most $\frac{2 \cdot 12}{3}=8$ vertices can be labelled by the colour 1 . A simple verification shows that $\rho_{1}(D)=8$.

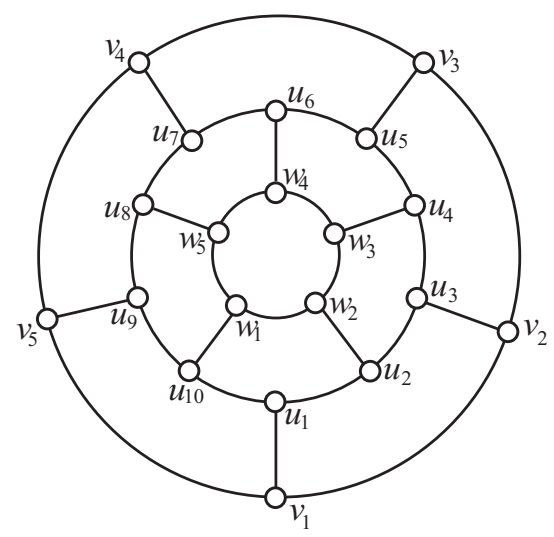

Fig. 3 A graph of the dodecahedron

We show that it is impossible to find a packing $k$ colouring of the graph $D$ with eight colours 1 and four colours 2. Let us try to assign eight vertices of $D$ in Fig. 3 the colour 1 . In such a case, every face contains the colour 1 exactly twice. Hence, two vertices of the 5cycle $C_{5}^{v}$ induced by $v_{1}, v_{2}, v_{3}, v_{4}$, and $v_{5}$ contain the colour 1. The same holds for the inner 5-cycle $C_{5}^{w}$ induced by $w_{1}, w_{2}, w_{3}, w_{4}$, and $w_{5}$. This immediately implies that exactly four vertices of the middle cycle $C_{10}^{u}$ induced by ten vertices $u_{1}, u_{2}, \ldots, u_{10}$ must be assigned the colour 1 . Without loss of generality, let $c\left(v_{1}\right)=1$. Only one of the vertices $v_{3}$ and $v_{4}$ can be assigned the colour 1 . Due to symmetry, both possibilities are equivalent. Let us consider $c\left(v_{3}\right)=1$. As, in this case, $c\left(v_{4}\right) \neq 1$ and $c\left(v_{5}\right) \neq 1$, the necessary assignment for the face with the vertices $v_{4}, v_{5}, u_{9}, u_{8}$, and $u_{7}$ on its boundary is $c\left(u_{7}\right)=c\left(u_{9}\right)=1$. Now, two other assignments the colour 1 on the cycle $C_{10}^{u}$ are possible only on the vertices $u_{2}, u_{3}$, and $u_{4}$ and therefore, $c\left(u_{2}\right)=c\left(u_{4}\right)=1$. For the 5-cycle $C_{5}^{w}$ we have $c\left(w_{1}\right)=c\left(w_{4}\right)=1$. A possible labelling of four vertices by the colour 2 forces that every 5 -cycle contains the colour 2 only once. Thus, this colour appears on the cycle $C_{10}^{u}$ exactly twice. For $c\left(v_{2}\right)=2$, only two of the vertices $u_{6}, u_{7}, u_{8}, u_{9}$, and $u_{10}$ can be assigned the colour 2. As $c\left(u_{7}\right)=c\left(u_{9}\right)=1$, the only possibility is $c\left(u_{6}\right)=c\left(u_{10}\right)=2$. In this case, none of the vertices of the cycle $C_{5}^{w}$ can be assigned the colour 2. For $c\left(v_{4}\right)=2$, $c\left(u_{3}\right)=c\left(u_{10}\right)=2$ is forced on the cycle $C_{10}^{u}$. But, in this case, in the inner cycle $C_{5}^{w}$ the only one vertex $w_{4}$ is in distance more than two from both $u_{3}$ and $u_{10}$. But, $c\left(w_{4}\right)=1$ and the colour 2 can be used at most three times 
again. The similar result is obtained also for the last possibility $c\left(v_{5}\right)=2$ in the outer cycle $C_{5}^{v}$. This implies that $\left|X_{1} \cup X_{2}\right| \leq 11$ in any case. Thus, as $\rho_{3}(D)=2, \rho_{4}(D)=2$, and $\rho_{i}(D) \leq 1$ for $i \geq 5$, at least five other colours $i \geq 5$ must be used and $\chi_{p}(D) \geq 9$. On the other hand, the packing 9-colouring $c\left(v_{1}\right)=c\left(v_{3}\right)=c\left(u_{2}\right)=c\left(u_{4}\right)=c\left(u_{7}\right)=$ $c\left(u_{9}\right)=c\left(w_{1}\right)=c\left(w_{4}\right)=1, c\left(v_{2}\right)=c\left(u_{6}\right)=c\left(u_{10}\right)=2$, $c\left(u_{3}\right)=c\left(w_{5}\right)=3, c\left(v_{4}\right)=c\left(w_{2}\right)=4, c\left(u_{1}\right)=5, c\left(v_{5}\right)=6$, $c\left(u_{8}\right)=7, c\left(u_{5}\right)=8$, and $c\left(w_{3}\right)=9$ confirms that $\chi_{p}(D) \leq$ 9. This completes the proof.

\section{PRISMS}

The $n$-side prism $H_{n}$, i.e. the $(4,4, n)$-polyhedron, $n \geq 3$, is a generalization of the cube. In the plane, it can be represented by the graph with the vertex set $V=\left\{u_{1}, u_{2}, \ldots, u_{n}, v_{1}, v_{2}, \ldots, v_{n}\right\}$ end the edge set $E=$ $\left\{\left\{u_{i}, u_{i+1}\right\} \cup\left\{v_{i}, v_{i+1}\right\} \cup\left\{u_{i}, v_{i}\right\}\right\}$, indices taken modulo $n$. The set of faces of $H_{n}$ consists of two $n$-gonal faces $\alpha=\left[u_{1} u_{2} \ldots u_{n}\right]$ and $\beta=\left[v_{1} v_{2} \ldots v_{n}\right]$ and $n$ quadrangles $\left[u_{i} u_{i+1} v_{i+1} v_{i}\right]$ for any $i=1,2, \ldots, n$, indices modulo $n$.

Lemma 3.1. Let $c$ be a packing $k$-colouring of the graph $H_{n}, n \geq 4$. For some $i$, let $c\left(u_{i}\right) \neq 1, c\left(u_{i+1}\right) \neq 1, c\left(v_{i}\right) \neq 1$, and $c\left(v_{i+1}\right) \neq 1$, indices taken modulo $n$. Then $k \geq 6$.

Proof. As none of the vertices $u_{i}, u_{i+1}, v_{i}$, and $v_{i+1}$ can be assigned a colour other than 1 more than once, $k \geq 5$. If the vertices $u_{i}, u_{i+1}, v_{i}$, and $v_{i+1}$ are assigned the colours 2 , 3,4 , and 5 , at least one of the vertices $u_{i-1}$ and $v_{i-1}$ or at least one of the vertices $u_{i+2}$ and $v_{i+2}$ must obtain a colour different from 1,2, 3, 4, and 5. Hence, at least six colours are necessary.

Lemma 3.2. Let $c$ be a packing $k$-colouring of the graph $H_{n}, n \geq 6$. For some $i$, let $c\left(v_{i-1}\right)=c\left(u_{i+1}\right)=1$ or $c\left(u_{i-1}\right)=$ $c\left(v_{i+1}\right)=1$, indices taken modulo $n$. Then $k \geq 6$.

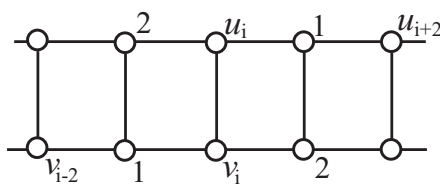

(a)

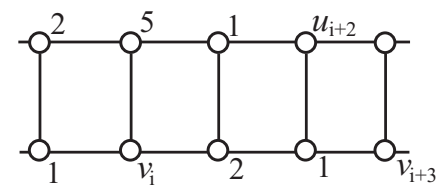

(b)

Fig. 4 The forced assignments of $H_{n}$

Proof. Without loss of generality, let $c\left(v_{i-1}\right)=c\left(u_{i+1}\right)=1$ and let $c$ be a packing $k$-colouring of the graph $H_{n}$ with $k \leq 5$. Assume first that some of the vertices $u_{i}$ and $v_{i}$, say $u_{i}$, is assigned by the colour 2 . In this case, three other colours 3, 4, and 5 must be used on the vertices $u_{i-1}, v_{i}$, and $v_{i+1}$. But, as $d\left(u_{i+1}, u_{i+2}\right)=1, d\left(u_{i}, u_{i+2}\right)=2$, and $d\left(x, u_{i+2}\right) \leq 3$ for $x \in\left\{u_{i-1}, v_{i}, v_{i+1}\right\}$, the vertex $u_{i+2}$ must obtain a colour different from all 1, 2, 3, 4, and 5. This forces that, for $k \leq 5, c\left(u_{i-1}\right)=c\left(v_{i+1}\right)=2$, because otherwise if only one vertex $u_{i-1}\left(v_{i+1}\right)$ is assigned by 2 , other three colours are necessary on the vertices $u_{i}, v_{i}$, and $v_{i+1}$ $\left(u_{i-1}, u_{i}\right.$, and $\left.v_{i}\right)$ and the vertex $v_{i-2}\left(u_{i+2}\right)$ needs one new colour. In Fig 4(a) it is easy to see that, for $c\left(u_{i-1}\right)=$ $c\left(v_{i+1}\right)=2, c\left(v_{i-2}\right) \notin\{1,2\}$ and $c\left(u_{i+2}\right) \notin\{1,2\}$. Moreover, as $d\left(v_{i-2}, u_{i+2}\right)=5$, the case $c\left(v_{i-2}\right)=5\left(c\left(u_{i+2}\right)=5\right)$ forces at least three other colours on the vertices $v_{i}, u_{i}$, and $u_{i+2}\left(u_{i}, v_{i}\right.$, and $\left.v_{i-2}\right)$. Hence, $c\left(u_{i}\right)=5$ or $c\left(v_{i}\right)=5$. Due to symmetry, assume $c\left(u_{i}\right)=5$. Now, in Fig. 4(b) it is possible to see that $c\left(v_{i}\right) \in\{3,4\}$ and $c\left(u_{i+2}\right) \in\{3,4\}$, which forces $c\left(v_{i+2}\right)=1$ and $c\left(v_{i+3}\right) \notin\{1,2,3,4,5\}$. So, at least six colours are necessary, which completes the proof.

Lemma 3.3. $\chi_{p}\left(H_{n}\right) \geq 5$ for $n \geq 9$.

Proof. Assume the vertices $u_{j}, u_{j+1}, u_{j+2}, v_{j}, v_{j+1}$, and $v_{j+2}$ of the graph $H_{n}$. If three of the considered vertices obtain the colour 1 , then at most one can be assigned the colour 2. On the other hand, if two of the vertices $u_{j}, u_{j+1}, u_{j+2}, v_{j}, v_{j+1}$, and $v_{j+2}$ obtain the colour 2 , then at most two other can be assigned the colour 1 . This forces at least two new colours on the vertices $u_{j}, u_{j+1}, u_{j+2}, v_{j}, v_{j+1}$, and $v_{j+2}$, which implies that $\chi_{p}\left(H_{n}\right) \geq 4$.

Without loss of generality, let $c\left(u_{i}\right)=4$. An assignment of the vertices $u_{i-1}, u_{i}, u_{i+1}, v_{i-1}, v_{i}$, and $v_{i+1}$ with only four colours requires that both colours 1 and 2 are used twice or the colour 1 is used three times. If the colour 2 is used twice, then $c\left(v_{i-1}\right)=c\left(u_{i+1}\right)=2$ or $c\left(u_{i-1}\right)=c\left(v_{i+1}\right)=2$. Without loss of generality, let $c\left(v_{i-1}\right)=c\left(u_{i+1}\right)=2$. Then, by Lemma 3.2, $c\left(u_{i-1}\right)=1$ and $c\left(v_{i}\right)=1$. So, $c\left(v_{i+1}\right)=3$ and at least one of the vertices $u_{i+2}$ and $v_{i+2}$ must be assigned the colour 5, see Fig. 5.a).

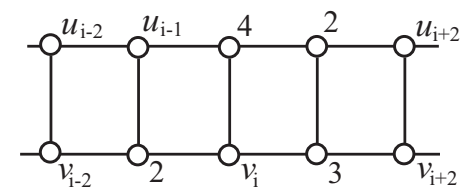

(a)

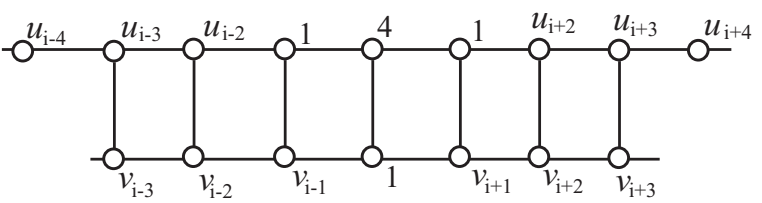

(b)

Fig. 5 The forced assignments of $H_{n}$ with only four colours

Thus, at least three of the vertices $u_{i-1}, u_{i+1}, v_{i-1}, v_{i}$, and $v_{i+1}$ are coloured by 1 and the unique possibility is shown in Fig 5(b). Now, it is easy to verify that on the vertices $v_{i-3}, \ldots, v_{i+3}, u_{i-4}, \ldots, u_{i+4}$, the colour 1 can be used at most seven times, the colour 2 at most four times, and the colour 3 at most three times. This, together with the fact that the colour 4 can be used only once, requires the fifth colour on the considered sixteen vertices. This completes the proof.

Lemma 3.4. Let $k \geq 5$ and let $c$ be a packing $k$-colouring of the graph $H_{n}, n \geq 6$, with $c\left(u_{i}\right)=5, c\left(v_{i}\right)=c\left(u_{i+1}\right)=1$, $c\left(v_{i+1}\right)=3$, and $c\left(v_{i-1}\right) \neq 4$, indices taken modulo $n$. Then for every positive integer $r$ there is a packing $k$-colouring of the graph $H_{n+6 r}$. 
Proof. In Fig. 6(a) there is a part of packing $k$-colouring of the graph $H_{n}$ with $c\left(u_{i}\right)=5, c\left(v_{i}\right)=c\left(u_{i+1}\right)=1, c\left(v_{i+1}\right)=$ 3 , and $c\left(v_{i-1}\right) \neq 4$. Let us insert the segment $F_{6}$ drawn in Fig. 66 b) by dotted lines between the edges $\left\{u_{i}, v_{i}\right\}$ and $\left\{u_{i+1}, v_{i+1}\right\}$. It is easy to verify that the resulting colouring of the graph $H_{n+6}$ is also packing $k$-colouring. The assignment of the last four vertices on the right side in Fig. 6(b) is in compliance with the assumption the Lemma. So, the inserting of the segment $F_{6}$ can be repeated, and the proof is done.

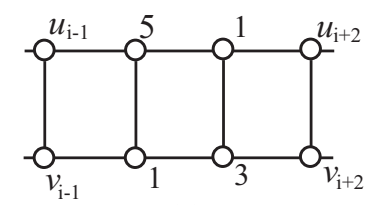

(a)

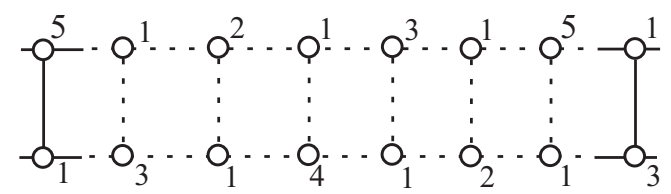

(b)

Fig. 6 The inserting of the segment $F_{6}$

Theorem 3.1. Let $H_{n}, n \geq 3, n \neq 4$, be the $n$-side prism. For $s=8,9,10, \ldots, \chi_{p}\left(H_{2 s}\right)=5$. Moreover, $\chi_{p}\left(H_{3}\right)=$ $\chi_{p}\left(H_{6}\right)=\chi_{p}\left(H_{10}\right)=\chi_{p}\left(H_{12}\right)=5$.

For $s=5,6,7, \ldots, \chi_{p}\left(H_{2 s+1}\right)=6$, and also $\chi_{p}\left(H_{5}\right)=$ $\chi_{p}\left(H_{14}\right)=6$.

$\chi_{p}\left(H_{7}\right)=\chi_{p}\left(H_{8}\right)=\chi_{p}\left(H_{9}\right)=7$.

Proof. In the proof, a packing $k$-colouring of the graph $H_{n}$ will be described by the matrix $C\left(H_{n}\right)$ with the colours $c\left(u_{i}\right), c\left(u_{i+1}\right), \ldots, c\left(u_{i+n-1}\right)$ of the vertices $u_{i}, u_{i+1}, \ldots$, $u_{i+n-1}$ in the first row and with the colours $c\left(v_{i}\right), c\left(v_{i+1}\right)$, $\ldots, c\left(v_{i+n-1}\right)$ of the vertices $v_{i}, v_{i+1}, \ldots, v_{i+n-1}$ in the second row, where the indices are taken modulo $n$. That is,

$$
C\left(H_{n}\right)=\left(\begin{array}{llll}
c\left(u_{i}\right) & c\left(u_{i+1}\right) & \ldots & c\left(u_{i+n-1}\right) \\
c\left(v_{i}\right) & c\left(v_{i+1}\right) & \ldots & c\left(v_{i+n-1}\right)
\end{array}\right) .
$$

First we describe the packing $k$-colourings of six graphs $H_{6}, H_{10}, H_{11}, H_{13}, H_{15}$, and $H_{20}$ which, together with Lemma 3.4 enable us to estimate the packing chromatic numbers for an infinite family of graphs $H_{n}$.

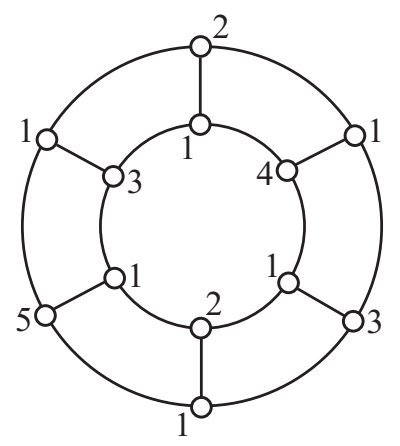

Fig. 7 A packing 5-colouring of $H_{6}$
For the graph $H_{6}$, the packing 5-colouring in Fig. 7 shows that $\chi_{p}\left(H_{6}\right) \leq 5$. This packing 5 -colouring can be described by the matrix

$$
C\left(H_{6}\right)=\left(\begin{array}{cccccc}
2 & 1 & 3 & 1 & 5 & 1 \\
1 & 4 & 1 & 2 & 1 & 3
\end{array}\right) .
$$

Similarly, from the next packing 5-colourings

$$
C\left(H_{10}\right)=\left(\begin{array}{llllllllll}
4 & 1 & 3 & 1 & 2 & 1 & 3 & 1 & 5 & 1 \\
1 & 2 & 1 & 5 & 1 & 4 & 1 & 2 & 1 & 3
\end{array}\right)
$$

and

$$
\begin{aligned}
& C\left(H_{20}\right)=\left(\begin{array}{llllllllll}
4 & 1 & 3 & 1 & 2 & 1 & 3 & 1 & 5 & 1 \\
1 & 2 & 1 & 5 & 1 & 4 & 1 & 2 & 1 & 3
\end{array}\right. \\
& \left.\begin{array}{llllllllll}
4 & 1 & 3 & 1 & 2 & 1 & 3 & 1 & 5 & 1 \\
1 & 2 & 1 & 5 & 1 & 4 & 1 & 2 & 1 & 3
\end{array}\right)
\end{aligned}
$$

of the graphs $H_{10}$ and $H_{20}$ we have $\chi_{p}\left(H_{10}\right) \leq 5$ and $\chi_{p}\left(H_{20}\right) \leq 5$. For the graphs $H_{11}, H_{13}$, and $H_{15}$, the packing 6-colourings

$$
\begin{aligned}
C\left(H_{11}\right) & =\left(\begin{array}{llllllll}
2 & 1 & 3 & 1 & 6 & 2 & 1 & 3 \\
1 & 4 & 1 & 2 & 5 & 1 & 4 & 1 \\
1 & 5 & 1 \\
2 & 1 & 3
\end{array}\right), \\
C\left(H_{13}\right) & =\left(\begin{array}{llllllll}
2 & 1 & 6 & 3 & 1 & 4 & 1 & 2 \\
1 & 4 & 2 & 1 & 5 & 1 & 3 & 1 \\
1 & 3 & 1 & 5 & 1 \\
6 & 1 & 2 & 1 & 3
\end{array}\right)
\end{aligned}
$$

and

$$
\begin{array}{r}
C\left(H_{15}\right)=\left(\begin{array}{llllllll}
2 & 1 & 3 & 1 & 6 & 1 & 2 & 5 \\
1 & 4 & 1 & 2 & 1 & 3 & 1 & 4 \\
1 & 3 & 1 & 4 & 1 & 5 & 1 \\
2 & 1 & 6 & 1 & 2 & 1 & 3
\end{array}\right)
\end{array}
$$

give $\chi_{p}\left(H_{11}\right) \leq 6, \chi_{p}\left(H_{13}\right) \leq 6$, and $\chi_{p}\left(H_{15}\right) \leq 6$. As all above packing $k$-colourings, $k=5,6$, satisfy the assumptions of Lemma 3.4 . for every non negative integer $r$ the inequalities $\chi_{p}\left(H_{6+6 r}\right) \leq 5, \quad \chi_{p}\left(H_{10+6 r}\right) \leq 5$, $\chi_{p}\left(H_{20+6 r}\right) \leq 5, \chi_{p}\left(H_{11+6 r}\right) \leq 6, \chi_{p}\left(H_{13+6 r}\right) \leq 6$, and $\chi_{p}\left(H_{15+6 r}\right) \leq 6$ are obvious .

The graph $H_{6}$ contains twelve vertices and, as the diameter of $H_{6}$ is four, $\rho_{2}\left(H_{6}\right)=\rho_{3}\left(H_{6}\right)=2$, and $\rho_{i}\left(H_{6}\right) \leq 1$ for all integers $i \geq 4$. As $\rho_{1}\left(H_{6}\right)=6$, every packing $k$ colouring of the graph $H_{6}$ requires at least $k=5$ colours. This, together with the packing 5-colouring in Fig. 7, confirms that $\chi_{p}\left(H_{6}\right)=5$. By Lemma 3.3. $\quad \chi_{p}\left(H_{n}\right) \geq 5$ for $n \geq 9$, which immediately implies that $\chi_{p}\left(H_{n}\right)=5$ for every even $n$ except of $n=8$ and $n=14$.

Eight vertices may obtain the colour 1 in a packing $k$ colouring of the graph $\mathrm{H}_{8}$. The diameter of the graph $\mathrm{H}_{8}$ is five, which implies that $\rho_{i}\left(H_{6}\right) \leq 1$ for $i \geq 5$. Morever, $\rho_{2}\left(H_{8}\right)=4, \rho_{3}\left(H_{8}\right)=\rho_{4}\left(H_{8}\right)=2$. It is easy to verify that $\left|X_{2} \cup X_{4}\right| \leq 3$ in a packing $k$-colouring with $\left|X_{1}\right|=\rho_{1}\left(G_{8}\right)=$ 8. Similarly, $\left|X_{1} \cup X_{4}\right| \leq 7$ if $\left|X_{2}\right|=4$ as well as $\left|X_{1} \cup X_{2}\right| \leq 9$ if $\left|X_{4}\right|=2$. This implies that in any packing $k$-colouring of the graph $H_{8}$ the inequality $\left|X_{1} \cup X_{2} \cup X_{4}\right| \leq 11$ holds. So, at most 13 vertices can be assigned the colours $1,2,3$, and 4. This requires at least three other colours and therefore, 
$\chi_{p}\left(H_{8}\right) \geq 7$. On the other hand, the reader can easy find a packing 7-colouring of the graph $H_{8}$. This proves that $\chi_{p}\left(H_{8}\right)=7$.

For the graph $H_{14}$ we have $\rho_{2}\left(H_{14}\right)=6, \rho_{3}\left(H_{14}\right)=4$, $\rho_{4}\left(H_{14}\right)=3$, and $\rho_{5}\left(H_{14}\right)=\rho_{6}\left(H_{14}\right)=2$. It is easy to verify that $\left|X_{1} \cup X_{2}\right| \leq 18$ in any packing $k$-colouring of the graph $H_{14}$. Thus, for the other ten vertices at least the colours $3,4,5$, and 6 are necessary. The packing 6colouring

$$
\begin{aligned}
C\left(H_{14}\right)=\left(\begin{array}{llllllll}
1 & 2 & 1 & 6 & 1 & 3 & 1 & 2 \\
4 & 1 & 3 & 1 & 2 & 1 & 5 & 1 \\
1 & 4 & 1 & 3 & 1 & 5 \\
3 & 1 & 6 & 1 & 2 & 1
\end{array}\right) &
\end{aligned}
$$

confirms that $\chi_{p}\left(H_{14}\right)=6$. Thus, the packing chromatic number $\chi_{p}\left(H_{n}\right)$ is given for all even $n$.

Now, let us turn to the graphs $H_{n}$ with odd $n$. We start with giving the packing chromatic number of the graphs $H_{n}$ for $n=3,5,7$, and 9. $\chi_{p}\left(H_{3}\right)=5$, because $\operatorname{diam}\left(H_{3}\right)=2$ and only the colour 1 can be used twice in a packing $k$ colouring of the graph $H_{3}$ with six vertices. As diam $\left(H_{5}\right)=$ $3, \rho_{1}\left(H_{5}\right)=4, \rho_{2}\left(H_{5}\right)=2$ and $\rho_{i}\left(H_{5}\right) \leq 1$ for $i \geq 3$. Thus, any packing $k$-colouring of ten vertices of the graph $H_{5}$ needs at least four colours other than 1 and 2 . Since there is a packing 6-colouring of $\mathrm{H}_{5}$, the packing chromatic number of the prism $H_{5}$ is six. The graph $H_{7}$ has fourteen vertices and diameter four. In a packing $k$-colouring of $H_{7}$, the colour 1 can be used at most six times. Moreover, $\rho_{2}\left(H_{7}\right)=3, \rho_{3}\left(H_{7}\right)=2$, and $\rho_{i}\left(H_{7}\right) \leq 1$ for $i \geq 4$. But, if $\left|X_{1}\right|=\rho_{1}\left(H_{7}\right)=6$, at most two vertices can be assigned by the colour 2 . This forces that the colours 1,2 , and 3 can appear on at most ten vertices and at least four other colours are necessary. A there is a packing 7-colouring of the graph $H_{7}, \chi_{p}\left(H_{7}\right)=7$. The colour 1 appears at most eight times in any packing $k$-colouring of the graph $H_{9}$ with eighteen vertices. Moreover, $\rho_{2}\left(H_{9}\right)=4, \rho_{3}\left(H_{9}\right)=2, \rho_{4}\left(H_{9}\right)=2$, and $\rho_{i}\left(H_{9}\right) \leq 1$ for $i \geq 5$. The reader can easy verify that $\left|X_{1} \cup X_{2}\right| \leq 11$ and therefore, the minimal number of used colours is seven and $\chi\left(H_{9}\right) \geq 7$. It is easy to find a packing 7-colouring of the graph $H_{9}$. This implies that $\chi_{p}\left(H_{9}\right)=7$.

For $s=5,6,7, \ldots$, the set of graphs $H_{2 s+1}$ is the same as the set of the graphs $H_{11+6 r}, H_{13+6 r}$, and $H_{15+6 r}$, $r=0,1,2, \ldots$. Thus, $\chi_{p}\left(H_{2 s+1}\right) \leq 6$ for all $s=5,6,7, \ldots$. It remains to show that for $s \in\{5,6,7, \ldots\}$, every packing $k$-colouring of the graph $\mathrm{H}_{2 s+1}$ requires at least six colours. By Lemma 3.3. $\chi_{p}\left(H_{2 s+1}\right) \geq 5$. To prove the inequality $\chi_{p}\left(H_{2 s+1}\right) \geq 6$ assume that, for some integer $s \geq 5$, there is a packing 5-colouring of the graph $H_{2 s+1}$. Among all such packing 5-colourings of the graph $\mathrm{H}_{2 s+1}$, consider a packing 5-colouring $c$ with maximal number of vertices labelled by the colour 1 . As the number $2 s+1$ is odd, there is a pair $u_{i}, u_{i+1}$ with $c\left(u_{i}\right) \neq 1$ and $c\left(u_{i+1}\right) \neq 1$, indices taken modulo $2 s+1$. In such a case, at most one of the vertices $v_{i}$ and $v_{i+1}$ can be assigned the colour 1 . In other words, $c\left(u_{j}\right) \neq 1$ and $c\left(v_{j}\right) \neq 1$ holds for at least one pair $u_{j}, v_{j}$. Lemma 3.1 forces that one vertex of both pairs $u_{j-1}, v_{j-1}$ and $u_{j+1}, v_{j+1}$ must obtain the colour 1. By Lemma 3.2, the possibilities $c\left(u_{j-1}\right)=c\left(v_{j+1}\right)=1$ or $c\left(v_{j-1}\right)=c\left(u_{j+1}\right)=$ 1 require at least six colours, a contradiction. Hence, $c\left(u_{j-1}\right)=c\left(u_{j+1}\right)=1$ or $c\left(v_{j-1}\right)=c\left(v_{j+1}\right)=1$. Without loss of generality assume that $c\left(u_{j-1}\right)=c\left(u_{j+1}\right)=1$. In this case, the vertex $v_{j}$ can be reassigned the colour 1 and, because of Lemma 3.3, the resulting colouring is a packing 5-colouring again. This contradiction with the assumption that $c$ is a packing 5-colouring of the graph $H_{2 s+1}$ with maximal number of colours 1 completes the proof.

\section{ACKNOWLEDGEMENT}

The research was supported by the Slovak VEGA grant No. 1/0309/11. This work was also supported by the Slovak Research and Development Agency under the contract No. APVV-0008-10.

The authors would like to thank the anonymous referees for several helpful comments and suggestions which improved the presentation of the paper.

\section{REFERENCES}

[1] ASHKINUZE, V. R.: On the number of semiregular polyhedra, Mat. Prosvesc. 1 (1957), 107-118. (In Russian)

[2] BREŠAR, B. - KLAVŽAR, S. - RALL, D. F.: On the packing chromatic number of Cartesian products, hexagonal lattices, and trees, Discrete Appl. Math. 155 (2007), 2303-2311.

[3] GODDARD, W. - HEDETNIEMI, S. M. - HEDETNIEMI, S. T. - HARRIS, J. M. - RALL, D. F.: Broadcast chromatic number of graphs, Ars Combin. 86 (2008), 33-49.

[4] HOLUB, P. - SOUKAL, R.: A note on packing chromatic number of the square lattice, Electronic Journal of Combinatorics 17 (2010), Note 17.

[5] FIALA, J. - GOLOVACH, P. A.: Complexity of the packing colouring problem for trees, Discrete appl. Math. 158 (2010), 771-778.

[6] FIALA, J. - KLAVŽAR, S. - LIDICKÝ, B.: The packing chromatic number of infinite product graphs, European. J. Combin 30 (2009), 1101-1113.

[7] JENDROL, S. - SCHRÖTTER, Š.: On rainbowness of semiregular polyhedra, Czechoslovak Mathematical Journal 58 (2008), 359-380.

Received July 23, 2012, accepted September 25, 2012

\section{BIOGRAPHIES}

Marián Klešč is associated professor at the Department of Mathematics and Theoretical Informatics, Faculty of Electrical Engineering and Informatics, Technical University of Košice. He received his $\mathrm{PhD}$. degree in discrete mathematics in 1995 at P. J. Šafárik University in Košice. His scientific research is focusing on topological graph theory and crossing numbers problems.

Štefan Schrötter is assistant professor at the Department of Mathematics and Theoretical Informatics, Faculty of Electrical Engineering and Informatics, Technical University of Košice. He received his CSc. degree in numerical mathematics in 1986 at Comenius University in Bratislava. At present, his research is focusing on problems in graph theory. 